\title{
Energy-Momentum Cathodoluminescence Spectroscopy of Dielectric Nanostructures
}

Sandro Mignuzzi, ${ }^{* \dagger}{ }^{\dagger}$ Mónica Mota, ${ }^{\dagger}$ Toon Coenen, ${ }^{\dagger, \S}$ Yi Li, ${ }^{\dagger}$ Andrei P. Mihai, ${ }^{\| \odot ~ P e t e r ~ K . ~ P e t r o v, " ~}$ Rupert F. M. Oulton, ${ }^{\dagger}$ Stefan A. Maier, ${ }^{\dagger, \perp}$ and Riccardo Sapienza ${ }^{\dagger}$

${ }^{\dagger}$ The Blackett Laboratory, Department of Physics, Imperial College London, London SW7 2BW, United Kingdom

${ }^{\ddagger}$ Delmic B.V., Kanaalweg 4, 2628 EB Delft, The Netherlands

${ }^{\S}$ Center for Nanophotonics, AMOLF, Science Park 104, 1098 XG Amsterdam, The Netherlands

"Department of Materials, Imperial College London, London SW7 2BP, United Kingdom

${ }^{\perp}$ Chair in Hybrid Nanosystems, Faculty of Physics, Ludwig-Maxilimians-Universität München, 80799 München, Germany

Supporting Information

ABSTRACT: Precise knowledge of the local density of optical states (LDOS) is fundamental to understanding nanophotonic systems and devices. Complete LDOS mapping requires resolution in energy, momentum, and space, and hence a versatile measurement approach capable of providing simultaneous access to the LDOS components is highly desirable. Here, we explore a modality of cathodoluminescence spectroscopy able to resolve, in single acquisitions, the dispersion in energy and momentum of the radiative LDOS. We perform measurements on a titanium nitride diffraction grating, bulk molybdenum disulfide, and silicon to demonstrate that the technique can probe and disentangle the dispersion of coherent and incoherent cathodoluminescence signals. The approach presented raises cathodoluminescence spectroscopy to a versatile tool for subwavelength design and optimization of nanophotonic devices in the reciprocal space.

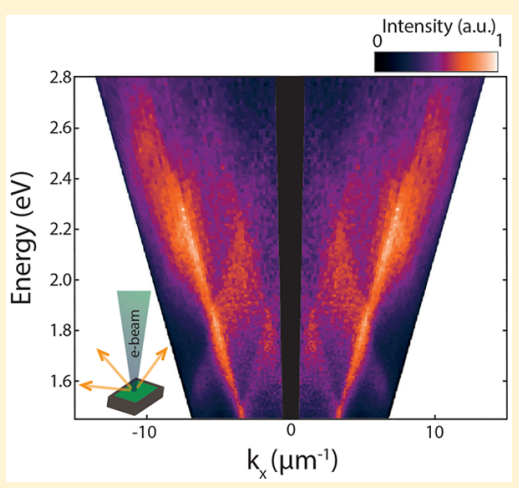

KEYWORDS: cathodoluminescence spectroscopy, local density of optical states, energy-momentum spectroscopy, dielectrics,

Fourier imaging

$\mathrm{P}$ hotonics developments have leveraged the arising opportunities of nanotechnology to boost the understanding of light - matter interactions on the nanoscale. While the nanophotonics field evolves, understanding and engineering light emission and absorption, which is ruled by the local density of optical states (LDOS), become increasingly relevant. Besides the first spatial maps, ${ }^{1,2}$ spectroscopy of the LDOS in reciprocal space has shed light onto the engineered optical modes even when confined to subwavelength volumes. A notable example is that of optical nanoantennas, which reshape and steer the radiation into specific emission angles (or specific momenta), through near-field interactions. ${ }^{3}$ This is particularly true for periodic structures such as dielectric photonic and plasmonic crystals, ${ }^{4,5}$ where light propagation is governed by the Bloch-Floquet theorem as well as for dielectric and plasmonic metasurfaces, ${ }^{6,7}$ both of which are characterized by strong wavevector dispersion.

Optical momentum-spectroscopy capable of energy-momentum imaging has been used to investigate the angular distribution of light by quantum emitters coupled to nanoantennas, but with a major restraint being imposed by the diffraction limit, along with the inherent experimental challenges to deterministically position the emitters. ${ }^{8}$ Conversely, scanning probe techniques such as scanning near-field optical microscopy can map the LDOS with a $\sim 100 \mathrm{~nm}$ spatial resolution typically, ${ }^{9}$ but usually lack spectral tunability, and the range of light momenta that can be collected is limited by the aperture of the probe.

Meanwhile, electron-based techniques such as cathodoluminescence (CL) spectroscopy are gaining increasing interest. ${ }^{10}$ Swift electrons interacting with matter give rise to a variety of optical excitations that can be leveraged to assess the optical and electronic properties of materials with nanometer spatial resolution. Traditionally spread across several areas of material science, CL spectroscopy has enabled in-depth investigations of electronic processes in semiconductors ${ }^{11}$ and, more recently, has made significant contributions to the field of nanophotonics, as a means to probe the radiative LDOS. ${ }^{4,12-14}$

When $\mathrm{keV}$ electrons travel across two different dielectrics, light is emitted in the form of far-field radiation, coherently with the evanescent field associated with the high-energy electrons. ${ }^{10}$ It has been recently suggested that, among the distinct coherent emission processes that can occur, transition radiation (TR) maps the vectorial component of the radiative LDOS along the electron trajectory, ${ }^{4,10}$ as opposed to energy

Received: November 21, 2017

Published: January 23, 2018 
a

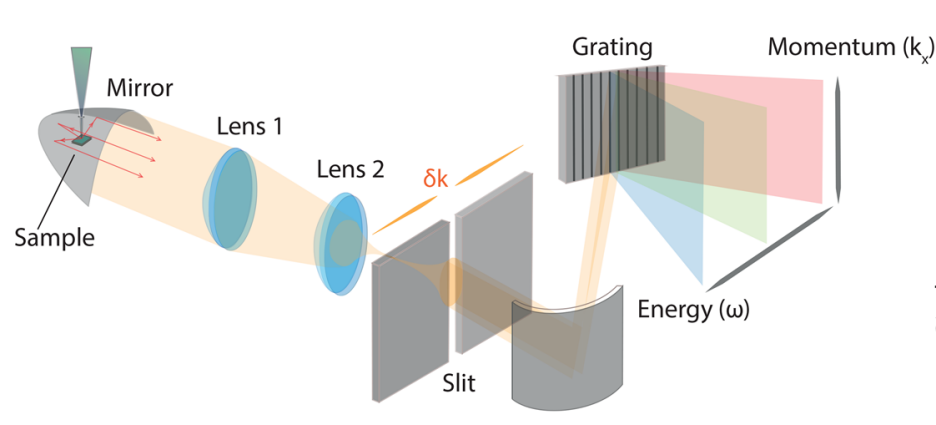

b Slit open $\cdots \cdots \cdots, C C D$

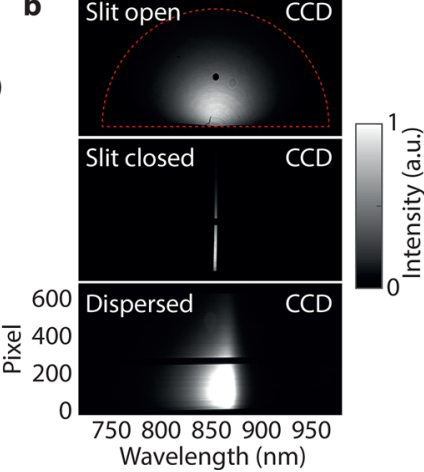

Figure 1. Experimental setup. (a) The sample is excited by an electron beam $(30 \mathrm{keV})$ and the CL emission is collimated by a parabolic mirror (NA $=0.98)$. In the optical path, the Fourier pattern is imaged on the entrance slit of the spectrometer. The slit selects the momenta in the direction of the parabola axis of the mirror. The light is dispersed by a grating on a CCD. (b) CCD images acquired from a bulk monocrystalline GaAs substrate with an open slit (top panel), a closed slit (middle panel), and a closed slit in the presence of the grating (bottom panel). The CL emission is dominated by band-edge emission from the GaAs.

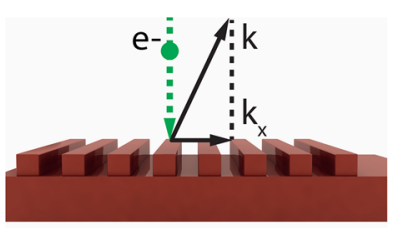

b

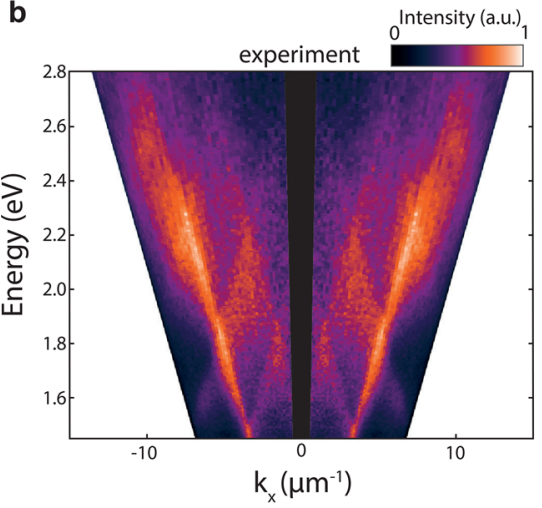

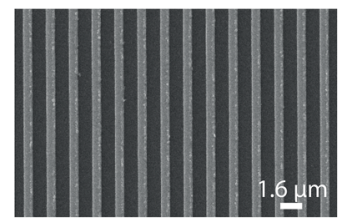

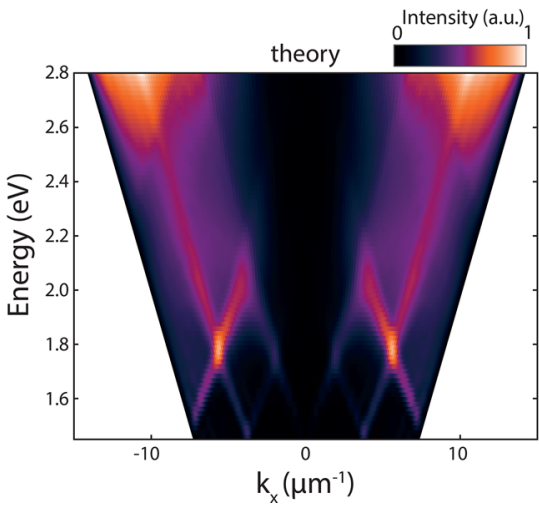

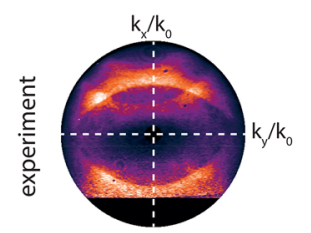
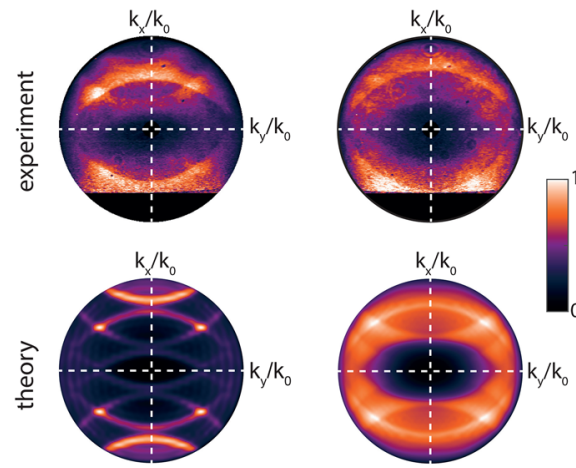

d
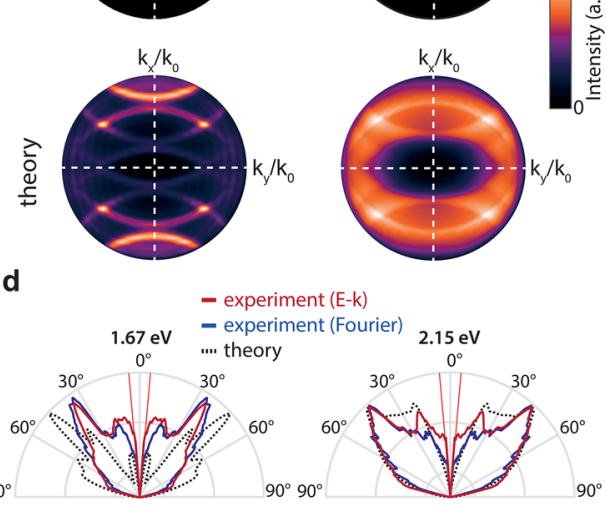

Figure 2. Energy-momentum dispersion measurements on a titanium nitride grating. (a) Sketch of the CL light emission process from a grating (SEM image on the right), where $\mathbf{k}$ is the light momentum vector and $k_{x}$ the momentum component perpendicular to the grooves. (b) Experimental (left) and simulated (right) cathodoluminescence intensity in the energy-momentum space. (c) Simulated and experimental distribution of CL intensity in momentum space at 1.67 and $2.15 \mathrm{eV}$. (d) Experimental angular patterns at 1.67 and $2.15 \mathrm{eV}$ as taken from the energy-momentum measurements shown in (b) and the Fourier measurements (in the absence of the slit) shown in (c). Dashed lines correspond to the simulated angular patterns from the theoretical dispersion shown in (b).

loss spectroscopy, which probes the total LDOS along the electron trajectory. In dielectric photonic crystals and cavities, CL spectroscopy has been used to obtain the $\operatorname{LDOS}^{4}$ with spatial resolution down to $30 \mathrm{~nm}$. Another approach to CL spectroscopy has also been recently developed along these lines, with the purpose of deriving information in the angular (i.e., momentum) domain, to measure the plasmonic dispersion in plasmonic crystal cavities. ${ }^{15}$

In this Article, we demonstrate a CL spectroscopy configuration that allows to probe, for the first time in a single measurement, the energy-momentum dispersion of $\mathrm{CL}$ emission. The proposed approach enables measurements of the energy-momentum dispersion of the LDOS while making it possible to disentangle the contributions of coherent and incoherent radiation to the total CL emission. The technique may be instrumental to the LDOS characterization of photonics devices with peculiar light dispersion, such as hyperbolic metamaterials ${ }^{16}$ and topological photonic crystals. ${ }^{17}$ Nanomaterials science may also benefit due to the possibility to probe the dielectric properties of anisotropic materials at the nanoscale, one prominent example being that of $2 \mathrm{D}$ materials. ${ }^{18}$

\section{ENERGY-MOMENTUM SPECTROSCOPY SETUP}

$\mathrm{CL}$ excited by an electron beam $(30 \mathrm{keV})$ and emitted from the sample is collected inside a scanning electron microscope (SEM), collimated by a parabolic mirror (numerical aperture $\mathrm{NA}=0.98$ ), and redirected to an optical collection system and spectrometer, as schematically depicted in Figure 1a. The Fourier pattern $I(k)$ generated by the optical lenses in Figure 1a is imaged onto a one-dimensional slit opening, which is used to select a subset of momenta $\left(k_{1}, k_{2}\right)$ of the light reflected from the central part of the parabolic mirror. This corresponds to selecting light components with $\mathbf{k}$-vectors lying within the plane normal to the sample surface and containing the parabola axis. 


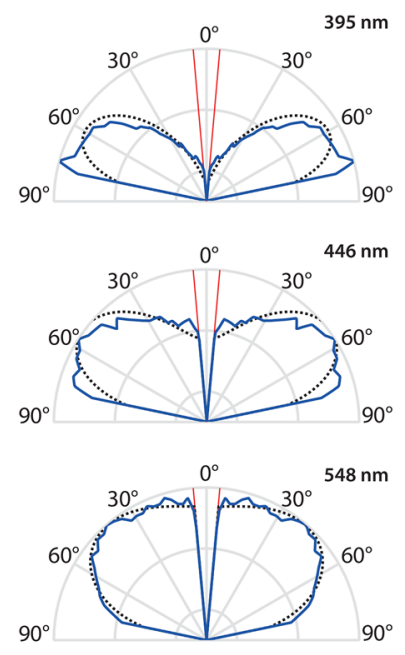

b

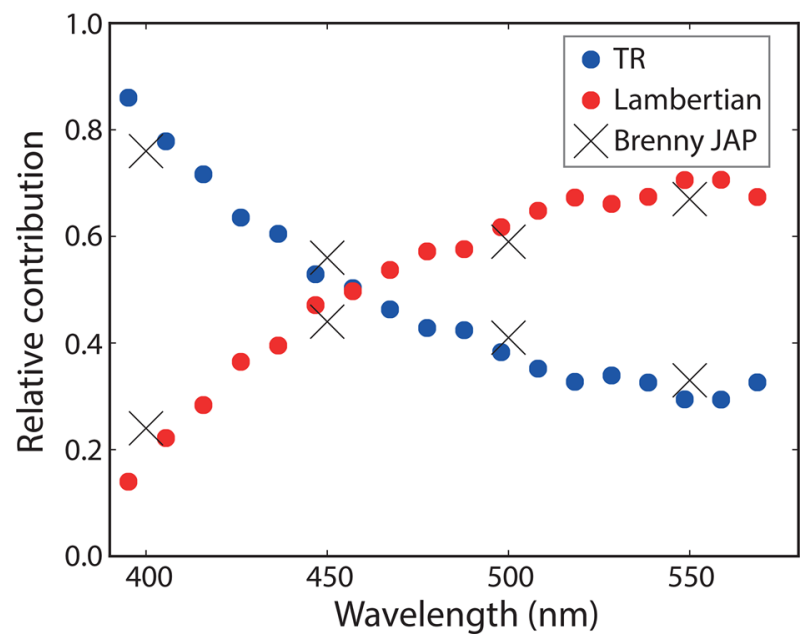

Figure 3. Angular patterns of silicon. (a) Experimental (continuous line) angular patterns from different wavelengths. The patterns are fitted (dashed line) using an incoherently summed combination of coherent (TR, transition radiation) and incoherent (Lambertian) contributions. (b) Relative contribution of TR and Lambertian components as a function of wavelength compared with ref 24, where Fourier spectroscopy, less resolved in wavelength space, is used instead.

A diffraction grating is then used to horizontally disperse the light on a two-dimensional CCD array, with the momentum components selected by the slit mapped in the vertical direction, yielding a $I(k, \lambda)$ map. This setup effectively implements momentum (also known as Fourier, or angleresolved) CL spectroscopy, whereby the angular distribution of the emitted light for a particular position on the sample is measured for specific emission wavelength bands. In order to retrieve the $\mathrm{CL}$ intensity as a function of energy and momentum, the image on the CCD is mapped onto the energy-momentum space, according to the specifics of the geometry and optical elements of the setup. This involves the transformation of angular coordinates to Cartesian coordinates on the $2 \mathrm{D}$ CCD pixel array and correction of intensity to take into account the solid angle covered per pixel. Figure $1 \mathrm{~b}$ shows the experimental CCD images collected from a bulk GaAs substrate, in three exemplifying configurations. When the slit is removed (top image), the Fourier pattern is imaged onto the CCD (for clarity, the outline of the mirror is marked with a dashed frame). Using the transformations relative to this specific geometry, the angle-resolved CL pattern can be then retrieved. In contrast, by inserting the slit in the optical path (middle image), a portion of light momenta is selected and subsequently dispersed in energy onto the CCD by means of a grating (bottom image).

In the following sections we focus on two key applications of energy-momentum CL spectroscopy, which is proven (i) to probe the energy-momentum dispersion of the LDOS in materials with dielectric function modulation in space $(\epsilon(r))$ and (ii) to probe the coherent and incoherent CL emission and their dependence on $\epsilon(\lambda)$.

\section{LDOS IN ENERGY-MOMENTUM SPACE}

Periodic structures with a tailored spatial variation of their dielectric properties can be used to modulate the LDOS and therefore to control the emission and the directivity of single emitters placed in proximity to, or embedded in, nanostructured materials. Notable examples are photonic nanostructures such as bullseyes ${ }^{19,20}$ and gratings. ${ }^{21}$ Here, we use a titanium nitride grating fabricated by sputtering on silicon and defined by electron beam lithography, with a periodicity of $1.665 \mu \mathrm{m}$, groove width of $0.665 \mu \mathrm{m}$, film thickness of $45 \mathrm{~nm}$, and groove thickness of $60 \mathrm{~nm}$. The corresponding SEM image is shown in the right panel of Figure 2a, while the left panel schematically depicts the CL emission process as a result of an electron impinging perpendicularly to the grating surface. This specific material choice is motivated by the fact that titanium nitride is particularly relevant for plasmonics at high temperatures. $^{22,23}$ In our experimental configuration, the spatial modulation of the grating is directed along the direction of collimation of the parabolic mirror (i.e., the $x$-axis). Note that in most SEMs the sample stage can rotate, which means that any azimuthal angle can be accessed experimentally through sample rotation. Scanning the Fourier pattern over the slit using (additional) optics might provide another means to fully map the momenta captured by the paraboloid mirror (one possible solution would be to translate lens 2 in Figure 1). The left panel of Figure $2 b$ outlines the experimental results obtained for the energy-momentum dispersion of the grating. This appears highly structured, with multiple crossovers of periodic series of local energy maxima and minima. This is a consequence of the nature of the angular radiation profile originating from the grating, which is composed of multiple lobes developing along various angles, as opposed to the conventional dipolar radiation pattern that would normally be observed at a planar interface between two dielectric media, as shown in Figure 3. Such lobes correspond to the grating diffraction orders whose separation in momentum space is $2 \pi / d$, with $d$ being the period of the grating. The measurement was performed above $1.4 \mathrm{eV}$, where the real part of the permittivity of TiN is positive, hence excluding the excitation of surface plasmon polaritons (SPPs) at the air-TiN interface. ${ }^{23}$ To gain insight into the nature of the grating light dispersion, numerical simulations have been carried out using the finite difference time domain (FDTD) method (further details are provided in the Methods section). We computed the far-field emission of a vertical dipole placed on the surface of the grating and positioned in the center of a groove. The simulated light dispersion presented in the right panel of Figure $2 \mathrm{~b}$ captures the complex evolution of the energy-momentum relation, showing remarkable agreement 


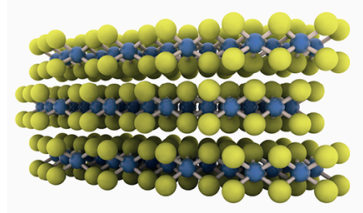

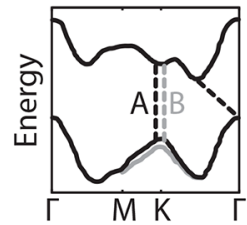

b

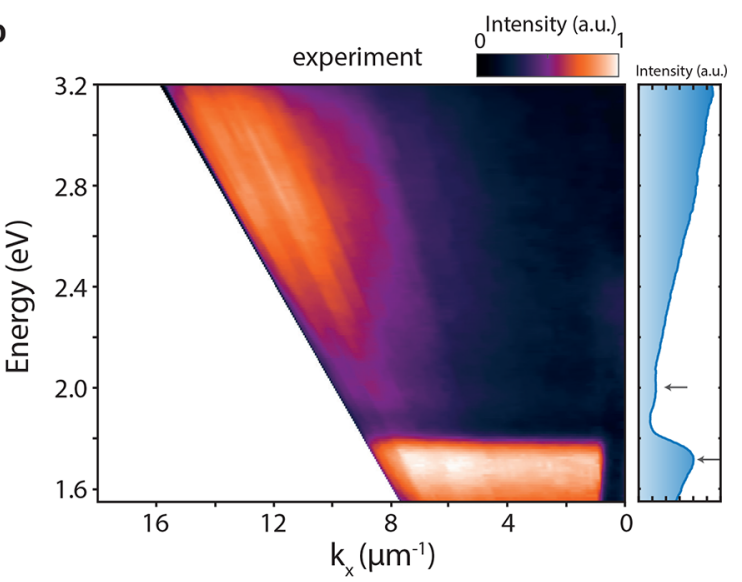

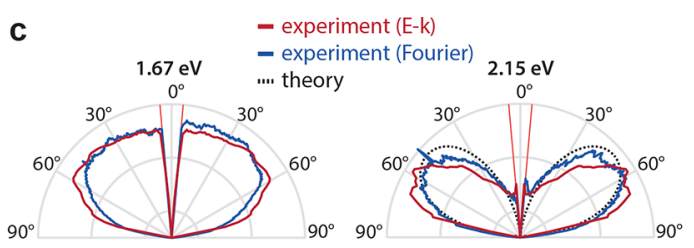

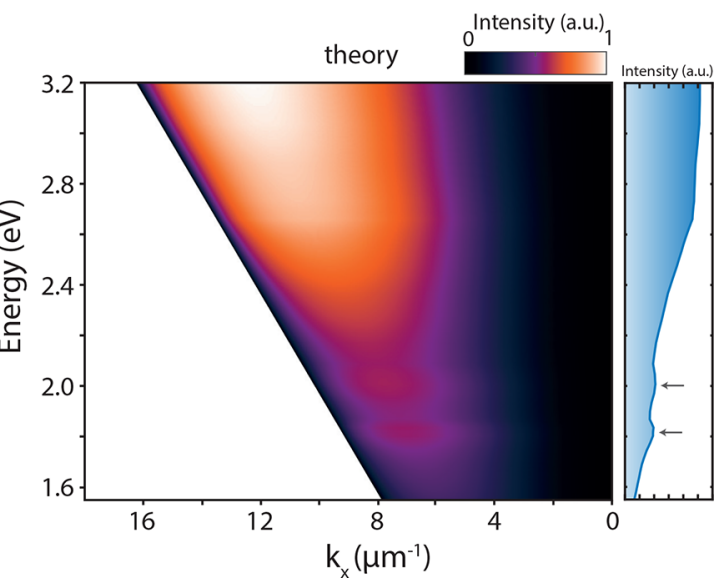

Figure 4. Energy-momentum dispersion of bulk $\mathrm{MoS}_{2}$. (a) The atomic structure (left) and a sketch of electronic dispersion (right) of MoS 2 , where the direct (A and B) transitions are marked with dashed lines. A possible indirect transition is also shown (discussed in the Supporting Information). (b) Experimental (left) and theoretical (right) energy-momentum dispersion and the energy spectrum, showing the TR signal (coherent emission). The luminescence from the direct transition (incoherent emission) is weakly dependent on the momentum $\left(k_{x}\right)$ and only visible in the experimental data. (c) Experimental angular patterns at 1.67 and $2.15 \mathrm{eV}$ as taken from the energy-momentum measurements shown in (b) as indicated by the arrows and Fourier measurements (in the absence of the slit). The theoretical TR pattern at $2.15 \mathrm{eV}$ is also shown by the dashed line.

with the experimental results. In Figures $2 \mathrm{c}$, d we report the isoenergy plot of the CL intensity in the $k_{x} / k_{0}$ and $k_{y} / k_{0}$ domains (where $k_{0}=2 \pi / \lambda$ ), measured at 1.67 and $2.15 \mathrm{eV}$ (to obtain these maps we used band-pass filters with a $15 \mathrm{~nm}$ line width). This approach essentially corresponds to performing angularresolved CL spectroscopy. Structured circular patterns arise in the spectra, corresponding to the iso-energy cut of the light dispersion, periodically shifted from the origin by $\lambda / d$ along the $k_{x} / k_{0}$ direction. The experimental maps feature a notable resemblance to the FDTD results presented in the bottom panel of Figure 2c. The angle-resolved CL spectroscopy maps are particularly useful because they provide in a single acquisition of $1-3 \mathrm{~s}$ a full energy-momentum distribution of the CL (Figure 2b). Moreover, in Figure 2d, the iso-energy profiles acquired with the two techniques, using a band-pass filter and the full energy-momentum mapping, are compared as well as with the theoretical angular patterns (dashed lines) taken from the simulated dispersion calculated in Figure $2 b$, with very good agreement.

\section{COHERENT AND INCOHERENT OPTICAL EXCITATIONS}

CL can originate from coherent and incoherent optical excitations, depending on whether they are phase related to the source, i.e., with the electromagnetic field of the electron passing through the material. TR is a coherent excitation and can be a measure of the LDOS. However, at the same time, energetic electrons can also excite electron-hole pairs for a given material, which then recombine, giving rise to luminescence, an incoherent process. Disentangling the two contributions within a CL energy spectrum is not a straightforward task; however, because these processes have different dispersion behaviors in momentum space, they can be resolved by energy-momentum spectroscopy. The TR originating from a simple interface between two dielectric media with different dielectric properties features a typical "doughnut-like" spatial far-field distribution. On the contrary, for a structured material, TR can become highly dispersive in the momentum domain, as observed for the aforementioned case of the titanium nitride grating. Incoherent processes are instead usually characterized by a Lambertian angular distribution of the emission profile, a consequence of the random orientation of the electron-hole pair recombination processes inside the excited material. These can be very efficient, and usually stronger than $\mathrm{TR}$, as the primary electron beam can generate a cascade of electrons, which, in turn, give rise to additional electron-hole pairs that recombine. We note that the incoherent signal from recombination, which originates at a depth of few hundred nanometers within the sample, can be scattered by a structured surface by far-field coupling only, as opposed to TR, which instead is generated at the sample surface, in the near field of the grating.

To further benchmark our technique, we study the energy evolution of the angular emission of a flat silicon surface using energy-momentum CL spectroscopy as compared to angleresolved CL spectroscopy used in ref 24 taken with a number of band-pass filters. We instead use energy-momentum CL spectroscopy to measure the wavelength dependence of the angular emission patterns. The resulting experimental angular evolution is shown in Figure 3a (blue solid lines) for three representative wavelengths of 395,446 , and $548 \mathrm{~nm}$. For shorter wavelengths, the angular pattern resembles that of a vertical dipolar emitter on a planar surface. The signal is dominated by the TR emission originating from electrons traversing free space into the silicon sample vertically. A weak Lambertian contribution is also present, which becomes 
progressively more dominant at longer wavelengths. The latter is associated with luminescence emission arising from the recombination of electron-hole pairs generated by inelastic events of high-energy electrons with the semiconductor. Such dispersive behavior can be explained by accounting for the fact that the TR intensity increases toward shorter wavelengths, whereas the luminescence is more likely to be reabsorbed for shorter rather than for longer wavelengths. ${ }^{24} \mathrm{We}$ model the silicon emission pattern using a linear combination of TR, as derived analytically, ${ }^{25}$ and Lambertian profiles. The weights of the linear combination, henceforth referred to as "relative contributions", are obtained by fitting the model to the experimental data and are plotted in Figure $3 \mathrm{~b}$. Their evolution as a function of the wavelength shows good agreement with previous measurements available in the literature, ${ }^{24}$ performed on silicon using angle-resolved CL spectroscopy. Remarkably, the superior resolution obtained with our technique enables access to finer details of the wavelength dependence and requires single-shot acquisitions, without relying on optical filters to select specific wavelength ranges.

Energy-momentum spectroscopy of bulk $\mathrm{MoS}_{2}$, an archetypal indirect gap semiconductor, allows us to separate coherent from incoherent optical excitations. Crystalline $\mathrm{MoS}_{2}$ has a layered atomic structure (schematically depicted in Figure 4a, left panel), which has attracted considerable interest in recent times, as the bulk crystal can be exfoliated to yield single, atomically thin layers. ${ }^{5}$ A sketch of the $\mathrm{MoS}_{2}$ electronic band structure is shown in the right panel of Figure 4a. The K-point of the Brillouin zone features a splitting of the valence band, which is instrumental to two possible direct transitions, known in the literature as A and B (black and gray vertical dashed lines in Figure 4b, respectively). Marked with an oblique black dashed line is the lowest energy transition, which is indirect in nature, between the valence valley at the $\Gamma$-point and the conduction valley at the Q-point.

The experimental map of the acquired energy-momentum dispersion is presented in the left panel of Figure $4 b$, along with the corresponding energy spectrum. In order to gain insight into these results, we performed an analytical calculation of the TR (Figure $4 b$, right panel) by using the experimental dielectric constant of $\mathrm{MoS}_{2}$ available in the literature (see Methods for further details). As also observed experimentally, the intensity of the TR is mainly governed by the $\sin ^{2} \theta \cos ^{2} \theta$ factor, which translates to a $\left(k_{x} / k_{0}\right)^{2}\left[1-\left(k_{x} / k_{0}\right)^{2}\right]$ dependence. In addition to this, two peaks at 1.8 and $2.0 \mathrm{eV}$ are also evident in the theoretical spectrum, originating from the dispersive behavior of the $\mathrm{MoS}_{2}$ dielectric constant, whose imaginary part is indeed characterized by two peaks at 1.8 and $2.0 \mathrm{eV}$, associated with the $\mathrm{A}$ and $\mathrm{B}$ transitions, respectively. In our experiment, we measure a peak at $2.0 \mathrm{eV}$, whereas we detect a dominant, asymmetric peak at $1.7 \mathrm{eV}$. Energy-momentum CL spectroscopy allows identifying the distinct nature of the 1.7 and $2.0 \mathrm{eV}$ spectral features by virtue of the fact that they feature different dispersion behaviors in the momentum domain. The $2.0 \mathrm{eV}$ peak is characterized by a $\left(k_{x} / k_{0}\right)^{2}\left[1-\left(k_{x} / k_{0}\right)^{2}\right]$ dependence, which is indicative of its TR nature associated with excitonic effects in the dielectric constant dispersion, whereas the $1.7 \mathrm{eV}$ feature follows a Lambertian evolution, i.e., $\sqrt{1-\left(k_{x} / k_{0}\right)^{2}}$; therefore we attribute it to incoherent luminescence processes, possibly due to the A transition.

However, we note that the A electronic transition is normally found $^{26,27}$ at $1.8 \mathrm{eV}$, whereas our measured luminescence signal is at $1.7 \mathrm{eV}$. We tentatively attribute this to self-absorption of luminescence by the $\mathrm{MoS}_{2}$ (higher energy luminescence is reabsorbed). ${ }^{11}$ Further observations shown in Figure $4 \mathrm{c}$ present the energy slices at 1.67 and $2.15 \mathrm{eV}$ of the angular patterns from the dispersion data in Figure $4 b$, indicating the two different coherent and incoherent regimes. The Supporting Information contains additional measurements performed in the spectral range where the indirect electronic transition manifests itself (marked with the oblique black line in Figure $4 a$, right panel), and we successfully detect the luminescence signal associated with it.

\section{CONCLUSIONS}

We have developed a cathodoluminescence spectroscopic technique able to directly measure the dispersion in energy and momentum space. In order to demonstrate its versatility and its functionality, we carried out CL studies on representative bulk and structured materials. We have shown that it allows disentangling the spectral signature of the transition radiation, which is a measure of the vertical component of the radiative LDOS, and luminescence signals due to electronic transitions, which are otherwise indistinguishable in a conventional energy spectrum. This is an important step toward full subwavelength characterization of the LDOS, which is of paramount importance for the design and optimization of nanoscale photonic devices.

\section{METHODS}

Sample Fabrication. Titanium nitride (TiN) nanogratings are fabricated with a chromium-based lift-off method. To eliminate the influence from the substrate, a $45 \mathrm{~nm}$ TiN thin film was first deposited onto p-Si (100) substrates by RF reactive magnetron sputtering, from a high-purity $\mathrm{Ti}$ (99.995\%) target (Pi-kem), using a MANTIS Deposition System at 800 ${ }^{\circ} \mathrm{C}$. The deposition was carried out using a gas mixture of $\mathrm{Ar}$ and $\mathrm{N}_{2}$ with a total constant pressure of $0.2 \mathrm{~Pa}$. To pattern periodic nanogratings with different widths and pitches, a 300 nm layer of poly(methyl methacrylate) (PMMA) $950 \mathrm{~K} \mathrm{~A} 4$ was coated at $3000 \mathrm{rpm}$ for $60 \mathrm{~s}$ and then baked for $3 \mathrm{~min}$ at 160 ${ }^{\circ} \mathrm{C}$. Nanostructures were then defined into PMMA by an electron beam exposure at $20 \mathrm{keV}$ (eLINE Plus, Raith $\mathrm{GmbH}$ ), followed by an MIBK/IPA (1:3)-based development procedure. The pattern was then cleaned by a plasma ash etch step (Femto, Diener Electronic GmbH, $12 \mathrm{~s}$ at $40 \%$ power). A 120 $\mathrm{nm}$ chromium layer was evaporated at $1.5 \AA$ /s via thermal evaporation (Amod, Angstrom Engineering Inc.), as the hard mask for a high-temperature sputter process, compatible with a standard acetone lift-off process. A second $60 \mathrm{~nm}$ thick $\mathrm{TiO}_{x} \mathrm{~N}_{y}$ thin film was deposited with exactly the same conditions. A standard chromium etchant containing ceric ammonium nitrate was used to remove the chromium mask and the $\mathrm{TiO}_{x} \mathrm{~N}_{y}$ residues on top of the mask. The sample was rinsed with DI water to complete the fabrication process.

Measurements. The measurements were performed in a SEM instrument (TESCAN) coupled to a CL system (SPARC, DELMIC, Holland). The electron beam was operated at 30 $\mathrm{keV}$ acceleration voltage and a beam current of $\sim 30 \mathrm{nA}$. Each energy-momentum CL measurement consists of a single exposure (integration time $180 \mathrm{~s}$ ) to the electron beam. The spectrometer (Andor Shamrock) was equipped with a grating (500 nm blaze, 150 grooves $/ \mathrm{mm}$ ) and a CCD camera (Andor iKon DU934P). The bare CCD image was properly trans- 
formed into the energy-momentum space, taking into account the parabolic shape of the mirror, the magnification of the optical path, and the camera pixel size. All the measurements were corrected by the instrument response using a single-crystal $\mathrm{Al}$ substrate as a reference. The signal originating from the flat Al substrate purely consists of $\mathrm{TR}$, which can be derived analytically. ${ }^{10,24}$ All the measurements were normalized to the instrument response, which was calculated by dividing the experimental $\mathrm{CL}$ intensity from the $\mathrm{Al}$ substrate to the theoretical value. All measurements were performed at room temperature.
Simulations and Theory. FDTD simulations for Figure 2 were carried out using the Lumerical FDTD Solutions package. A vertically aligned electrical point dipole is placed at a height of $5 \mathrm{~nm}$ above the material, and at the center of a groove in the case of the grating, and the emitted power is calculated as a function of angle and emission frequency. The refractive index of TiN is determined by ellipsometry. The theory in Figure 4 employs the following analytical expression for the energy radiated in the backward emission in terms of the differential spectrum per unit wavelength and solid angle: ${ }^{25}$

$$
\begin{aligned}
& \frac{\mathrm{d}^{2} N}{\mathrm{~d} \lambda \mathrm{d} \Omega}=\frac{\alpha \beta^{2}}{\pi \lambda} n_{1} \sin ^{2} \theta_{1} \cos ^{2} \theta_{1} \\
& \times\left|\frac{\left(\epsilon_{2}-\epsilon_{1}\right)\left(1-\beta^{2} \epsilon_{1}+\beta \sqrt{\epsilon_{2}-\epsilon_{1} \sin ^{2} \theta_{1}}\right.}{\left(1-\beta^{2} \epsilon_{1} \cos ^{2} \theta_{1}\right)\left(1+\beta \sqrt{\epsilon_{2}-\epsilon_{1} \sin ^{2} \theta_{1}}\right)\left(\epsilon_{2} \cos \theta_{1}+\sqrt{\epsilon_{2} \epsilon_{1}-\epsilon_{1}^{2} \sin ^{2} \theta_{1}}\right)}\right|^{2}
\end{aligned}
$$

where medium 1 and 2 represent air and $\mathrm{MoS}_{2}$ respectively, the electron velocity is $v=\beta c$, where $c$ is the speed of light in vacuum, $\alpha=1 / 137$ is the fine structure constant, and $\theta_{1}$ the emission angle. Here, we approximate the anisotropic dielectric tensor as the value of the in-plane dielectric constant, $\epsilon_{2}$, of $\mathrm{MoS}_{2}$ measured in the literature. ${ }^{28}$

\section{ASSOCIATED CONTENT}

\section{S Supporting Information}

The Supporting Information is available free of charge on the ACS Publications website at DOI: 10.1021/acsphotonics.7b01404.

Coordinate transformed CL spectroscopy energymomentum dispersion data for a titanium nitride film and bulk molybdenum disulfide (PDF)

\section{AUTHOR INFORMATION}

\section{Corresponding Author}

*E-mail: s.mignuzzi@imperial.ac.uk.

\section{ORCID $\odot$}

Sandro Mignuzzi: 0000-0001-6051-2022

Yi Li: 0000-0002-6134-3117

Andrei P. Mihai: 0000-0002-7204-9087

\section{Notes}

The authors declare the following competing financial interest(s): Toon Coenen is employee of Delmic BV, a company that has brought a cathodoluminescence system on the market based on a design developed at AMOLF and that was used in this work.

The data is publicly available in Figshare at DOI: 10.6084/ m9.figshare.c.3990738.

\section{ACKNOWLEDGMENTS}

S.M. and R.S. acknowledge funding by EPSRC (EP/P033369/ 1, EP/M027791/1). P.K.P. and A.P.M. acknowledge the support from the Henry Royce Institute through EPSRC grant EP/R00661X/1. S.A.M. acknowledges the EPSRC Reactive Plasmonics Programme (EP/M013812/1) and the Lee-Lucas Chair in Physics. The authors acknowledge Éric Piel (Delmic B.V.) for help with the acquisition software development.

\section{REFERENCES}

(1) Barnard, E. S.; Coenen, T.; Vesseur, E. J. R.; Polman, A.; Brongersma, M. L. Imaging the Hidden Modes of Ultrathin Plasmonic Strip Antennas by Cathodoluminescence. Nano Lett. 2011, 11 (10), $4265-4269$.

(2) Vesseur, E. J. R.; Coenen, T.; Caglayan, H.; Engheta, N.; Polman, A. Experimental Verification of $\mathrm{n}=0$ Structures for Visible Light. Phys. Rev. Lett. 2013, 110 (1), 1-5.

(3) Coenen, T.; Vesseur, E. J. R.; Polman, A.; Koenderink, A. F. Directional Emission from Plasmonic Yagi-Uda Antennas Probed by Angle-Resolved Cathodoluminescence Spectroscopy. Nano Lett. 2011, 11, 3779-3784.

(4) Sapienza, R.; Coenen, T.; Renger, J.; Kuttge, M.; van Hulst, N. F.; Polman, A. Deep-Subwavelength Imaging of the Modal Dispersion of Light. Nat. Mater. 2012, 11 (9), 781-787.

(5) Saito, H.; Yamamoto, N. Control of Light Emission by a Plasmonic Crystal Cavity. Nano Lett. 2015, 15 (9), 5764-5769.

(6) Jahani, S.; Jacob, Z. All-Dielectric Metamaterials. Nat. Nanotechnol. 2016, 11 (1), 23-36.

(7) Li, G.; Clarke, B. P.; So, J.-K.; MacDonald, K. F.; Zheludev, N. I. Holographic Free-Electron Light Source. Nat. Commun. 2016, 7, 13705.

(8) Curto, A. G.; Volpe, G.; Taminiau, T. H.; Kreuzer, M. P.; Quidant, R.; van Hulst, N. F. Unidirection Emission of a Quantum Dot Coupled to a Nanoantenna. Science 2010, 20, 930-933.

(9) Colas Des Francs, G.; Girard, C.; Weeber, J. C.; Dereux, A. Relationship between Scanning near-Field Optical Images and Local Density of Photonic States. Chem. Phys. Lett. 2001, 345 (5-6), 512516.

(10) García de Abajo, F. J. Optical Excitations in Electron Microscopy. Rev. Mod. Phys. 2010, 82 (1), 209-275.

(11) Yacobi, B. G.; Holt, D. B. Cathodoluminescence Scanning Electron Microscopy of Semiconductors. J. Appl. Phys. 1986, 59 (4), R110.1063/1.336491.

(12) Kuttge, M.; Vesseur, E. J. R.; Koenderink, A. F.; Lezec, H. J.; Atwater, H. A.; García de Abajo, F. J.; Polman, A. Local Density of States, Spectrum, and Far-Field Interference of Surface Plasmon Polaritons Probed by Cathodoluminescence. Phys. Rev. B: Condens. Matter Mater. Phys. 2009, 79 (11), 2-5.

(13) Fang, Y.; Verre, R.; Shao, L.; Nordlander, P.; Käll, M. Hot Electron Generation and Cathodoluminescence Nanoscopy of Chiral Split Ring Resonators. Nano Lett. 2016, 16 (8), 5183-5190.

(14) Atre, A. C.; Brenny, B. J. M.; Coenen, T.; García-Etxarri, A.; Polman, A.; Dionne, J. A. Nanoscale Optical Tomography with Cathodoluminescence Spectroscopy. Nat. Nanotechnol. 2015, 10 (5), 429-436. 
(15) Honda, M.; Yamamoto, N. Size Dependence of Surface Plasmon Modes in One-Dimensional Plasmonic Crystal Cavities. Opt. Express 2013, 21 (10), 11973-11983.

(16) Poddubny, A.; Iorsh, I.; Belov, P.; Kivshar, Y. Hyperbolic Metamaterials. Nat. Photonics 2013, 8 (1), 78-78.

(17) Rechtsman, M. C.; Zeuner, J. M.; Plotnik, Y.; Lumer, Y.; Nolte, S.; Segev, M.; Szameit, A. Photonic Floquet Topological Insulators. Nature 2012, 496 (7444), 196-200.

(18) Hu, D.; Yang, X.; Li, C.; Liu, R.; Yao, Z.; Hu, H.; Corder, S. N. G.; Chen, J.; Sun, Z.; Liu, M.; Dai, Q. Probing Optical Anisotropy of Nanometer-Thin van Der Waals Microcrystals by near-Field Imaging. Nat. Commun. 2017, 8 (1), No. 1471.

(19) Osorio, C. I.; Coenen, T.; Brenny, B. J. M.; Polman, A.; Koenderink, A. F. Angle-Resolved Cathodoluminescence Imaging Polarimetry. ACS Photonics 2016, 3 (1), 147-154.

(20) Davanço, M.; Rakher, M. T.; Schuh, D.; Badolato, A.; Srinivasan, K. A Circular Dielectric Grating for Vertical Extraction of Single Quantum Dot Emission. Appl. Phys. Lett. 2011, 99 (4), 97-100.

(21) Jun, Y. C.; Huang, K. C. Y.; Brongersma, M. L. Plasmonic Beaming and Active Control over Fluorescent Emission. Nat. Commun. 2011, 2, 283.

(22) Catellani, A.; Calzolari, A. Plasmonic Properties of Refractory Titanium Nitride. Phys. Rev. B: Condens. Matter Mater. Phys. 2017, 95 (11), 115145.

(23) Braic, L.; Vasilantonakis, N.; Mihai, A.; Villar Garcia, I. J.; Fearn, S.; Zou, B.; Alford, N. M. N.; Doiron, B.; Oulton, R. F.; Maier, S. A.; Zayats, A. V.; Petrov, P. K. Titanium Oxynitride Thin Films with Tunable Double Epsilon-Near-Zero Behavior for Nanophotonic Applications. ACS Appl. Mater. Interfaces 2017, 9 (3C5), 2985729862.

(24) Brenny, B. J. M.; Coenen, T.; Polman, A. Quantifying Coherent and Incoherent Cathodoluminescence in Semiconductors and Metals. J. Appl. Phys. 2014, 115.24430710.1063/1.4885426

(25) Yamamoto, N.; Araya, K.; Toda, A.; Sugiyama, H. Light Emission from Surfaces, Thin Films and Particles Induced by HighEnergy Electron Beam. Surf. Interface Anal. 2001, 31 (2), 79-86.

(26) Li, Y.; Chernikov, A.; Zhang, X.; Rigosi, A.; Hill, H. M.; Van Der Zande, A. M.; Chenet, D. A.; Shih, E. M.; Hone, J.; Heinz, T. F. Measurement of the Optical Dielectric Function of Monolayer Transition-Metal Dichalcogenides: $\mathrm{MoS}_{2}, \mathrm{MoSe}_{2}, \mathrm{WS}_{2}$, and $\mathrm{WSe}_{2}$. Phys. Rev. B: Condens. Matter Mater. Phys. 2014, 90 (20), 1-6.

(27) Qiu, D. Y.; da Jornada, F. H.; Louie, S. G. Optical Spectrum of $\mathrm{MoS}_{2}$ : Many-Body Effects and Diversity of Exciton States. Phys. Rev. Lett. 2013, 111 (21), 216805.

(28) Beal, A. R.; Hughes, H. P. Kramers-Kronig Analysis of the Reflectivity Spectra of $2 \mathrm{H}-\mathrm{MoS}_{2}, 2 \mathrm{H}-\mathrm{MoSe}_{2}$ and $2 \mathrm{H}-\mathrm{MoTe}_{2}$. J. Phys. C: Solid State Phys. 1979, 881, 881. 